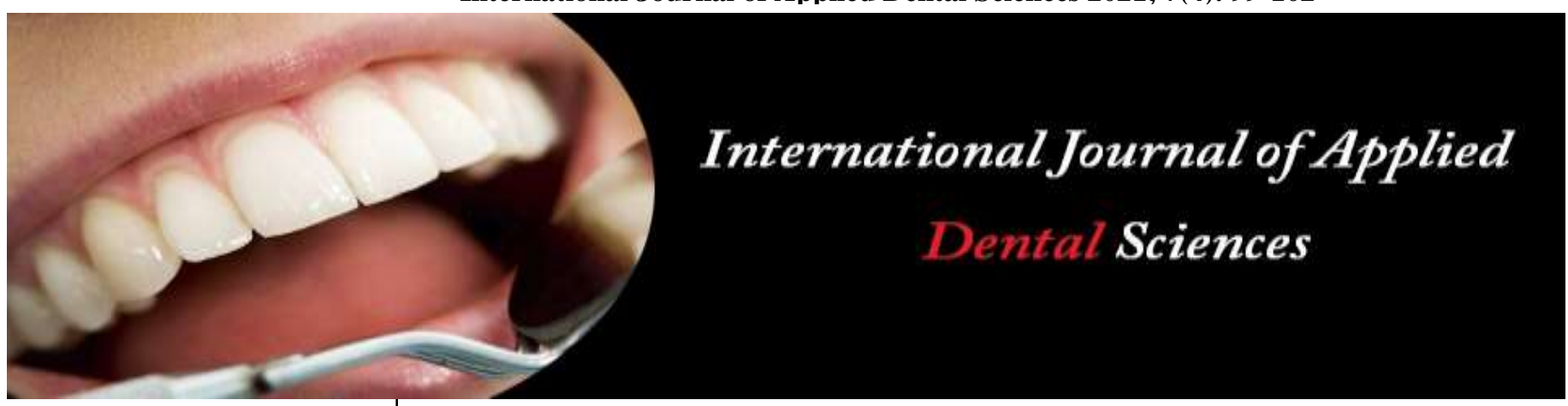

ISSN Print: 2394-7489

ISSN Online: 2394-7497

IJADS 2021; 7(4): 99-102

(C) 2021 IJADS

www.oraljournal.com

Received: 15-08-2021

Accepted: 19-09-2021

Dr. Aasmita K Kabade

Post Graduate Student,

Government Dental College and Hospital, Aurangabad,

Maharashtra, India

Dr. Kishor M Mahale

HOD, Professor and Guide,

Government Dental College

and Hospital, Aurangabad,

Maharashtra, India

Dr. Shankar P Dange

Dean and Professor, Government

Dental College and Hospital,

Aurangabad, Maharashtra, India

Dr. Smita A Khalikar

Professor, Government Dental

College and Hospital,

Aurangabad, Maharashtra, India

Corresponding Author:

Dr. Aasmita K Kabade

Post Graduate Student,

Government Dental College and Hospital, Aurangabad,

Maharashtra, India

\section{A modified impression technique for flabby and severely atrophic mandibular ridge: A case report}

\author{
Dr. Aasmita K Kabade, Dr. Kishor M Mahale, Dr. Shankar P Dange and \\ Dr. Smita A Khalikar
}

DOI: $\underline{\text { https://doi.org/10.22271/oral.2021.v7.i4b.1358 }}$

\section{Abstract}

Complete denture fabrication in clinically compromised situations such as flabby ridge and/ or atrophic ridge is a challenging task for the prosthodontist for years. Flabby ridge and highly resorbed residual mandibular ridge is commonly observed in older patients. Accurate impressioning of these tissues plays a major role in ensuring a well-fitting and stable prosthesis. This article describes a technique for making a definitive impression for highly displaceable and resorbed residual ridges. The technique is especially applicable for mandibular edentulous ridges. The choice of the impression materials, as well as the design of the impression tray, focuses on preventing distortion of the displaceable residual ridges during impression making.

Keywords: Flabby tissue, severely atrophic mandibular residual ridges, impression technique

\section{Introduction}

Complete denture prosthodontics will remain an important part of dental education and practice. The performance of a complete denture often depends on basic principles of impression making. Recording the entire functional denture-bearing area ensures maximum support, retention and stability for the denture during use. However, difficulties arise when the quality of the denture-bearing areas are not suitable for this purpose ${ }^{[1]}$.

'Flabby' alveolar ridges and/or severely resorbed ridges pose significant problems for the provision of stable and retentive dental prostheses for affected patients. Flabby ridge is a superficial area of mobile soft tissue affecting the maxillary or mandibular alveolar ridges. It can develop when hyperplastic soft tissue replaces the alveolar bone and is a common finding. Masticatory forces can displace this mobile denture-bearing tissue, leading to altered denture positioning and loss of peripheral seal. Forces exerted during the act of impression taking can result in distortion of the mobile tissue. The resulting stability of the denture can be poor. In the soft tissue, varying amounts of metaplastic cartilage and/or bone have been reported [2]. The lesser resilient tissues can displace the dentures under masticatory load leading to loss of peripheral seal causing poor retention of the denture ${ }^{[3]}$.

Management of a flabby ridge is mainly by three approaches:

1. surgical removal of fibrous tissue prior to conventional prosthodontics,

2. implant retained prosthesis
a) fixed,
b) removable,

3. conventional prosthodontics without surgical intervention ${ }^{3}$

Each technique has its advantages and shortcomings. The advantage of the surgical technique is that it provides a firm denture bearing area. Its limitations include chances of decrease in vestibular height. It is contraindicated in patients who are unwilling to undergo a surgical treatment. A particular problem is encountered in the conventional impression making if a flabby ridge is present within a denture bearing area. If the flabby tissue is compressed during conventional impression making it will later tend to recoil and dislodge the overlying denture [3]. 
Residual ridge resorption is considered as a multi-factorial, biomechanical disease that results from a combination of anatomic, metabolic and mechanical determinants. According to Glossary of prosthodontic terms ninth edition, residual ridge resorption is defined as a term used for the diminishing quantity and quality of the residual ridge after the teeth are extracted. Residual ridge resorption is a chronic, progressive, irreversible and cumulative disease. It is our main concern as a prosthodontist to incorporate proper impression techniques and measures to minimize the residual ridge resorption in the treatment plan.

Many authors have suggested different impression techniques to overcome unfavorable consequences of flabby tissues and some authors have given several impression techniques for atrophied ridges to minimize further resorption. So, in this article an effort has been made to improve stability of mandibular denture by combining various techniques to obtain an accurate impression of flabby tissue and atrophied mandibular ridge.

\section{Case report}

A 63 year old female patient reported to department of prosthetics of Government Dental College and Hospital, Aurangabad with a chief complaint of replacement of missing teeth with no significant medical history. Patient had lost her all mandibular teeth and maxillary anterior teeth 1 year ago and she extracted her upper posterior teeth 1 month back as they were mobile and she was completely edentulous since 1 month.

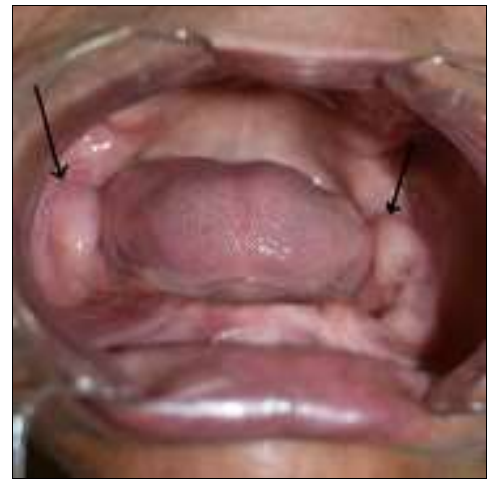

Fig 1: Showing excessively flabby retromolar tissue on both sides with severely atrophied residual ridge

On intraoral examination patient had completely edentulous upper and lower arches. Maxillary ridge has incompletely healed extracted sockets whereas severely resorbed mandibular ridge. One of the major finding was excessively flabby retromolar tissue on the both sides.

For incompletely healed maxillary extraction socket patient was advised massaging with a gum paint along with warm saline gargle and asked to report after one week. The treatment options of implant supported prosthesis and surgical excision of the flabby tissue were suggested to the patient. But the patient was not willing to undergo any surgical procedures so it was decided that upper and lower complete dentures will be fabricated with a modified impression technique.
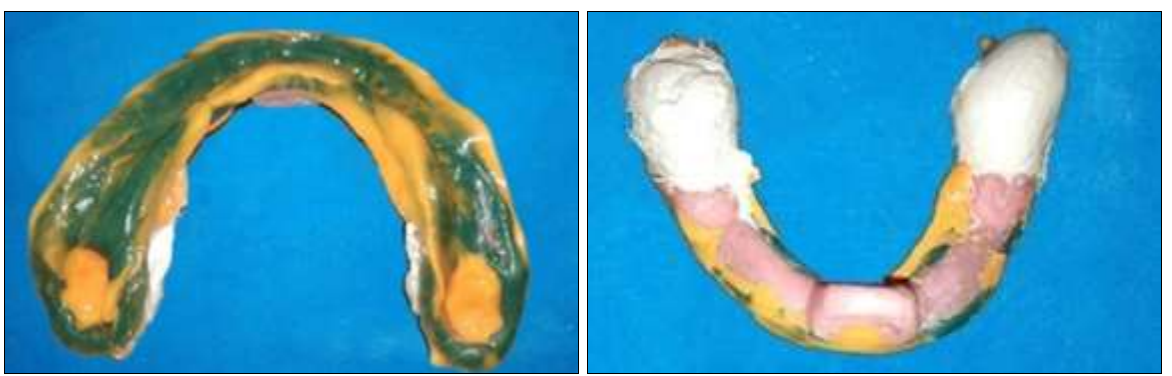

Fig 2, 3: Showing modified impression technique for flabby and severely atrophic mandibular ridge with windows over retromolar flabby tissue

1. Maxillary primary impression was made using medium fusing impression compound (Y Dents, MDM Corporation, Delhi). And mandibular primary impression was made with admix technique. The impressions were poured and the displaceable tissues were identified on the mandibular cast.

2. For maxillary, spacer was designed according to J.J Sharry's design and for mandible, cast was first adapted with a layer of wax to provide extra relief in the flabby region (retromolar tissue) followed by adaptation of spacer over relieving area leaving buccal self-area.

3. Custom trays were made using autopolymerising acrylic resin. Maxillary border moulding was done using low fusing type -1 impression compound (DPI, Mumbai) and wash impression was made with light body condensation silicone impression material (Zetaplus, Zhermack).

4. Mandibular impression was made with all green technique. Green stick compound (DPI, Mumbai) is kneaded to a homogenous mass and is loaded on the special tray and border movements were carried out.

5. Intraorally flabby tissue was marked and those markings were transferred to made impression. Window was created which corresponds to both retromolar flabby tissue on the made impression.

6. Final wash impression of entire ridge and flabby retromolar tissue was made simultaneously with light body condensation silicone impression material (Zetaplus, Zhermack). Light body impression material was applied over entire intaglio surface of the impression along with peripheral borders, simultaneously light body impression material was injected over retromolar flabby tissue and then wash impression was taken simultaneously by performing all functional movements.

7. When the light body impression material was set, it was supported with gauze soaked in dental plaster. Impression was removed from mouth carefully. Impression was inspected for its completeness. Stability of impression was checked by asking the patient to perform functional movements of tongue.

8. Master cast was poured in dental stone. Jaw relations with proper vertical and centric were recorded. For the try in appointment waxed denture were first tried for occlusion and esthetics, followed by fabrication of conventional complete denture by compression moulding technique. 


\section{Discussion}

Management of flabby tissue along with resorbed ridges is a cumbersome and difficult task especially in mandible because of smaller denture bearing area and other anatomical limitations. Surgical management requires patient cooperation and may not be feasible at all times. Alternatively, prosthetic management of such flabby tissues and severely resorbed ridges involves special impression techniques mainly to achieve stability [4]. Various techniques have been recommended for management of flabby tissue and for severely resorbed ridges. The clinical choice of selecting impression technique has fallen mainly to personal preference, based on analysis of theoretical principles ${ }^{[2]}$.

For management of flabby tissue, there are various techniques given by different authors like “Zafrulla Khan's Window technique [5]", "Palatal splinting using a two-part tray system" and "Two part impression technique" by Osborne ${ }^{[1]}$, One part impression technique (Selective perforation tray) [2], Controlled lateral pressure technique ${ }^{2}$ etc. Modifications can be done in spacer design by using double spacer to provide extra relief over flabby area or modifications can be done in custom tray design according to amount of displaceable tissue. Where distortion of displaceable tissue is minimal, the use of perforations of the special tray overlying the fibrous region may be all that is required. Where distortion of displaceable tissue is significant, window can be created corresponding to flabby tissue so that final wash impression of those tissue can be recorded in most stable and undisplaced position. The use of holes, windows and wax relieve reduces the pressure and minimize the displacement of the bearing tissues.

For management of severely resorbed ridges many authors have given various techniques like, "modified fluid wax impression technique" by kian et al. ${ }^{[9]}$, "Green All Compound Technique" by Tunkiwala and Ram ${ }^{[11]}$, "Admixed technique" by McCord and Tyson ${ }^{[10]}$ etc. The case presented in this article has severely resorbed residual ridge and extremely flabby retromolar tissue on both the sides. Thus to record resorbed ridge in its functional form and to further reduce its residual resorption as well as to record flabby tissue in its static position to minimize its displacement, a modified impression technique has been used.

In this technique, the primary impression was recorded using admixed technique due to its lower compressibility and better flow characteristics. Spacer design covered crest of residual ridge to minimize stresses on them leaving the buccal shelf area. Extra relief was given over both the retromolar flabby tissue so that it should be recorded in most stable position. Low fusing impression compound was used to selectively exert pressure, by restricting the flow of impression material in the stress bearing area and scraping out the material from non-stress bearing areas and also from flabby retromolar areas. Retromolar tissue was extremely flabby, thus window technique was chosen to record it. Final impression was made using light body polyvinyl siloxane impression material. In a study conducted by Al-Ahmad et al. ${ }^{[12]}$ it was found that low viscosity polyvinyl siloxane material exhibited least pressure and provided an excellent record of minute details of the residual ridge in its passive form.

The goal of this technique is to provide maximum stability covering maximum denture bearing area, selectively distributing the pressure. This is attributed to various steps contributing to impression making. First, the intimate contact of custom tray helps in distributing maximum forces to the buccal shelf area. This is achieved by the spacer design.
Second, the viscosity of the low fusing impression compound removes any soft tissue folds and smoothens them over the mandibular bone, thus reducing the potential for discomfort arising from the "atrophic sandwich" of the mucosa between the denture and the bone. And window technique that minimizes distortion of unsupported flabby tissue. The sequencing of the clinical steps in this modified physiologic impression technique was done considering the principles of "Admixed technique" by McCord and Tyson and "Green all compound technique" by Tunkiwala and Ram and "window technique" by Zafrulla Khan. The technique described in this article prevents sandwich of atrophic mucosa, prevents distortion of flabby tissue, thus maintains the health and integrity of mucosa and residual ridge and renders comfort to the patient during denture usage. Further, contemporary and readily available impression materials are used in this technique. The drawbacks of this technique include discomfort due to heat used during functional molding with green stick impression compound. Furthermore, the brittleness of the material during scraping is also a disadvantage. Besides, its usefulness as a new technique needs to be evaluated in more cases.

Choice of treatment modality is made by keeping in mind that the requirement of stability and retention of the prosthesis must be balanced along with the preservation of the health of oral tissues for every patient.

\section{Conclusion}

Prosthodontic rehabilitation of a patient with unfavorable tissues and ridge in a conventional manner is a difficult task. Modifications in the treatment procedures should be considered to fulfil the patient's functional and esthetic demands. The modified impression technique described in this article can be used in situations like presence of flabby tissue as well as severely atrophic mandibular ridge. This technique can give good results in terms of stability of the denture if performed accurately.

\section{References}

1. Jayaprakash MB, Sahu K, Khan M, Khoriya S, Jadhav S, Kendre B et al. Management of Flabby Ridge Cases: A Challenge in Clinical Practice. Int J Adv Health Sci 2014;1(5):32-37.

2. Crawford RWI, Walmsley AD. A review of prosthodontic management of fibrous ridges. British Dental Journal 2005;199:715-719.

3. Umesh Pai Y, Vikram Simha Reddy, Rushad Nariman Hosi. A Single Step Impression Technique of Flabby Ridges Using Monophase Polyvinylsiloxane Material: A Case Report. Case Reports in Dentistry Volume 2014, 6. Article ID 104541.

4. Daniel S, Daniel AY, Kurian N. A modified physiologic impression technique for atrophic mandibular ridges. CHRISMED J Health Res 2017;4:204-8.

5. Khan Z, Jaggers J, Shay J. Impressions of unsupported movable tissues. JADA 1981;103:590-92.

6. Labban N. Management of the flabby ridge using a modified window technique and polyvinylsiloxane impression material. Saudi Dental Journal 2017.

7. Shinto Thomas et al. Complete denture impression techniques for resorbed ridges: a review. The journal of prosthetic and implant dentistry 2019;2(3):124-128.

8. Smita Sara Manoj, Vidya Chitre, Meena Aras. Management of Compromised Ridges: A Case Report. J Indian Prosthodont Soc 2011;11(2):125-129. 
9. Kian Tan M, Michael Singer T, Radi Masri, Carl Driscoll F. Modified fluid wax impression for a severely resorbed edentulous mandibular ridge. J Prosthet Dent 2009; 101:279-282.

10. McCord JF, Tyson KW. A conservative prosthodontic option for the treatment of edentulous patients with atrophic (flat) mandibular ridges. $\mathrm{Br}$ Dent $\mathrm{J}$ 1997;182:469-472.

11. Tunkiwala A, Ram S. Management of mandibular poor foundation: Conventional complete dentures. Dent Pract 2013;11:34-7.

12. Al-Ahmad A, Masri R, Driscoll CF, Von Fraunhofer J, Romberg E. Pressure generated on a simulated mandibular oral analog by impression materials in custom trays of different design. $\mathbf{J}$ Prosthodont 2006;15:95-101. 